\title{
A study on edge marking scheme of various standard edge detectors
}

\author{
Shaifali Pande \\ Department of Electronics and \\ Communication Engineering \\ National Institute of Technology \\ Agartala, India -799055
}

\author{
Vivek Singh Bhadouria \\ Department of Electronics and \\ Communication Engineering \\ National Institute of Technology \\ Agartala, India -799055
}

\author{
Dibyendu Ghoshal \\ Department of Electronics and \\ Communication Engineering \\ National Institute of Technology \\ Agartala, India -799055
}

\begin{abstract}
This paper presents a study on edge marking scheme of various standard edge detectors viz. Sobel, Prewitt, Roberts, Laplacian of Gaussian (LoG) and Canny. On the basis of edge map obtained for a synthetic rectangular board image, obtained from five edge detectors, suggests classifying the detectors in three categories namely, pre-marker, post-marker and mixed-marker. Pratt's figure of merit (PFOM) is used as a quantitative evaluation criterion for the above mentioned classification. Experimental results obtained for Lena and Parrot image provides convincing results to establish proposed classification.
\end{abstract}

\section{General Terms}

Image Processing

\section{Keywords}

Edge detection, Pratt's figure of merit.

\section{INTRODUCTION}

Edge detection is a fundamental process in image segmentation technique which is performed to determine the edges or lines in an image and outline them in a proper way. The main aim of edge detection is to classify the image data according to the sharp intensity changes in an image resulting from discontinuities in depth or surface orientation, variation in scene illumination etc. The performance measure for the edge detection is how well edge detector markings match with the visual perception of object boundaries [1]. The detection process is carried out by the examination of local intensity changes at each pixel element of an image.

A number of edge detectors have been proposed such as Sobel, Roberts, Marr-Hildreth, DoG (Difference of Gaussian) etc. which is either based on finding the first order derivative maxima or minima or zero crossings in the second order derivatives of pixel intensity in an image. The second order derivative based edge detectors are highly sensitive to noise and leads to error in edge detection in presence of noise [2] and therefore seldom used in practical applications. Three major kinds of errors [3] associated with edge detection are 1) missing accurate edge points, 2) non-localized edges and 3) noise fluctuations considered as edge points.

In this paper, an attempt has been made to study the edge marking scheme of five standard edge detectors viz Sobel, Prewitt, Roberts, LoG (Laplacian of Gaussian) and Canny. Pratt's Figure of Merit (PFOM) is used here as an assessment criterion for the standard edge detectors using test images of a synthetic rectangular board image, Lena and Parrot image. The five edge detectors are considered commutatively as ideal edge map to evaluate PFOM.
The organization of the paper is as follows. An introduction to the standard edge detectors considered in this paper is given in section II. Section III follows with an overview to PFOM. Section IV focuses on the implementation of the detectors on the test images and their experimental results to demonstrate the marking scheme for various edge detectors along with the quantitative evaluation with the help of PFOM. Finally, the conclusions are drawn in section $\mathrm{V}$.

\section{EDGE DETECTORS}

\subsection{Sobel Operator}

The Sobel operator is a discrete differentiation operator which computes the gradient for the intensity changes at each point in an image. The operator consists of two $3 \times 3$ kernels as shown in figure 1 .
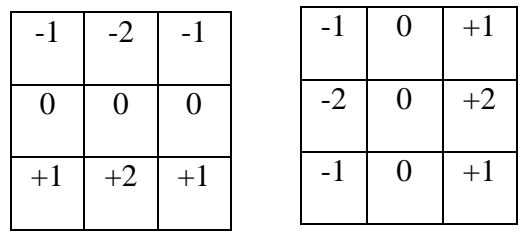

Fig 1: The 3x3 kernels for the Sobel operator

\begin{tabular}{|l|l|l|}
\hline $\mathrm{P}_{1}$ & $\mathrm{P}_{2}$ & $\mathrm{P}_{3}$ \\
\hline $\mathrm{P}_{8}$ & $\mathrm{P}_{0}$ & $\mathrm{P}_{4}$ \\
\hline $\mathrm{P}_{7}$ & $\mathrm{P}_{6}$ & $\mathrm{P}_{5}$ \\
\hline
\end{tabular}

Fig 2: The 3x3 region centered on pixel $P_{0}$ for a source image $I(x, y)$

Masks which are symmetric across the center point are often useful for computation of edge direction and hence this operator is easier to implement. Considering $I(x, y)$ as the source image and using the $3 \times 3$ region centered on pixel $\mathrm{P}_{0}$, (figure 2) the two kernels are convolved separately with the original image to obtain the approximations of the derivatives or the gradient components, each for the horizontal and vertical edge orientations (say $\mathrm{G}_{\mathrm{x}}$ and $\mathrm{G}_{\mathrm{y}}$ ). The magnitude for the gradient is computed as

$$
|G|=\sqrt{G_{x}^{2}+G_{y}^{2}}
$$

and the angle of orientation of the gradient is given as 


$$
\alpha(x, y)=\arctan \left(\frac{G_{y}}{G_{x}}\right)
$$

The Sobel operators are preferred because they have better noise suppression (smoothing) characteristics [2].

\subsection{Roberts Cross-Gradient Operator}

The Roberts cross-gradient operator proposed by Lawrence Roberts in 1965 [4] is one of the earliest edge detector to use 2-D masks for diagonal edge detection. The operator computes the gradient of an image through discrete differentiation, achieved by calculating the sum of the squares of the differences between diagonally adjacent pixels. Consider the $3 \times 3$ region in figure 2 , each square representing a pixel, the spatial gradient measurement based on implementing diagonal differences through Roberts operator of an image $I(x, y)$, is given as:

$$
g_{x}=\frac{\partial I}{\partial x}=\left(p_{5}-p_{0}\right)
$$

and

$$
g_{y}=\frac{\partial I}{\partial y}=\left(p_{6}-p_{4}\right)
$$

These derivatives are implemented to perform edge detection through Roberts operator with the following two kernels shown in figure 3 .

\begin{tabular}{|c|c|}
\hline+1 & 0 \\
\hline 0 & -1 \\
\hline-1 & 0 \\
\hline-1 & +1 \\
\hline
\end{tabular}

Fig 3: The 2x2 kernels for Roberts operator

These kernels can be applied separately to the input image where $G_{x}$ and $G_{y}$ are the points in an image formed by convolving with the first kernel and the second kernel, respectively. The magnitude of the gradient is then given by

$$
|G|=\sqrt{G_{x}^{2}+G_{y}^{2}}
$$

and the direction of gradient is defined by the angle

$$
\alpha(x, y)=\arctan \left(\frac{G_{y}}{G_{x}}\right)
$$

\subsection{Canny Edge Detector}

In the areas where edge quality is of superior interest, the Canny edge detector is a promising alternative to the edge detectors discussed above. The Canny operator was developed by John F. Canny in 1986 [5] whose approach is based on the three objectives for an 'optimal' edge detector: a) low error rate, b) Edge points should be well localized and c) only one response to single edge point. In order to satisfy the preceding objectives Canny suggested that a good approximation to the optimal edge detector is the first derivative of Gaussian.

The Canny edge detection algorithm uses a multi stage algorithm in order to detect a wide range of edges in images The algorithm constitutes the following basic steps:

1. Filter the noise and smooth the input image through Gaussian filter.
2. Find the edge strength by computing the gradient magnitude and angle of gradient vector for edge direction.

3. Apply non-maxima suppression to the gradient magnitude to trace along the edge direction and suppress any pixel value that is not considered to be an edge and give a thin line to the input image.

4. Use double thresholding or hysteresis [6] and connectivity analysis [2] to detect and connect edges.

If the single threshold point for edge detection is set too low or too high, there will be either false positives or false negatives edges, respectively. Canny's algorithm proposed to improve the situation of false edge point detection by using hysteresis thresholding, using two thresholds: a low threshold and a high threshold. The Canny detector is found to be more complex to implement and requires more execution time as compared to other edge detector mentioned above, however it is found to perform better than other operators [7].

\subsection{The Laplacian of Gaussian (LoG) Operator}

The Laplacian operator highlights the regions of rapid intensity changes in an image and hence is often used as an edge detection operator. As the Laplace of an image detects the noise along with the edges in an image, the image is smoothened first by convolving by a 2-D Gaussian kernel of standard deviation $\sigma$

$$
G(x, y)=e^{-\frac{x^{2}+y^{2}}{2 \sigma^{2}}}
$$

The expression for LoG is then given by

$$
\nabla^{2} G(x, y)=\left[\frac{x^{2}+y^{2}-2 \sigma^{2}}{\sigma^{4}}\right] e^{-\frac{x^{2}+y^{2}}{2 \sigma^{2}}}
$$

The LoG operator is then convolved with the input image, say $I(x, y)$, as

$$
g(x, y)=\left[\nabla^{2} G(x, y)\right] * I(x, y)
$$

The above $2 \mathrm{D}$ LoG expression can be represented by a $5 \times 5$ kernel approximation in figure 4 .

\begin{tabular}{|c|c|c|c|c|}
\hline 0 & 0 & 1 & 0 & 0 \\
\hline 0 & 1 & 2 & 1 & 0 \\
\hline 1 & 2 & -16 & 2 & 1 \\
\hline 0 & 1 & 2 & 1 & 0 \\
\hline 0 & 0 & 1 & 0 & 0 \\
\hline
\end{tabular}

Fig 4: The 5x5 kernel for LoG operator

The kernels of any size can be approximated by using the above expression for LoG. The edge detection in an image using LoG operator can thus be obtained by the following steps:

1. Apply LoG to the input image.

2. Detect the zero-crossings of the image.

3. Apply thresholding to minimize the weak zerocrossings caused due to noise. 
The relative importance of zero-crossing through LoG is an active area of research [8-9].

\subsection{Prewitt Operator}

The Prewitt operator [10] is a discrete differentiation operator which functions similar to the Sobel operator, by computing the gradient for the image intensity function. As compared to Sobel, the Prewitt masks are simpler to implement [2] but are very sensitive to noise [7] [11].

The operator uses two $3 \times 3$ size masks which gives more information regarding the direction of the edges as they consider the nature of data on the opposite sides of the center point of the mask. The two masks are convolved with the original image to obtain the approximations of derivatives for the horizontal and vertical edge changes, separately. The approximations of gradient obtained using Prewitt operator are expected to be more accurate than that of the Roberts which uses masks of size $2 \times 2$ [2]. The mask used to calculate the gradient at the point $\mathrm{P}_{0}$ are shown in figure 5 .

\begin{tabular}{|c|c|c|}
\hline-1 & -1 & -1 \\
\hline 0 & 0 & 0 \\
\hline+1 & +1 & +1 \\
\hline
\end{tabular}

\begin{tabular}{|c|c|c|}
\hline-1 & 0 & +1 \\
\hline-1 & 0 & +1 \\
\hline-1 & 0 & +1 \\
\hline
\end{tabular}

Fig 5: The 3x3 kernels of Prewitt operator

The magnitude and directions of the gradient can be given as

$$
\begin{gathered}
|G|=\sqrt{G_{x}^{2}+G_{y}^{2}} \\
\alpha(x, y)=\arctan \left(\frac{G_{y}}{G_{x}}\right)
\end{gathered}
$$

where $G_{x}$ and $G_{y}$ are the two images of the same size as the original image and represents the horizontal and vertical approximations of gradient at each point, respectively.

\section{PRATT'S FIGURE OF MERIT (PFOM)}

Pratt [12] introduced a figure of merit to analyze and balance the associated errors in edge detection process. Mathematically, PFOM is given as

$$
R=\frac{1}{I_{N}} \sum_{i=1}^{I_{A}} \frac{1}{1+a d^{2}}
$$

Where $I_{N}=\max \left\{I_{I}, I_{A}\right\}$ and $I_{I}$ and $I_{A}$ are the number of ideal and actual edge map points, respectively, $d$ is the distance of separation of an actual edge point normal to a line of ideal edge points and $a$ is the scaling constant. In the present work, the scaling function, $a$ is considered as $1 / 9$ (following the Pratt's original work).

The separation distance, $d$ plays a vital role in the evaluation of figure of merit. From equation 1, it can be seen that the figure of merit is inversely proportional to the distance $d$. For a smeared edge, the distance $d$ between ideal and actual edge map increases and consequently the value of rating factor $R$ is reduced. As a result, the edge detector which results in localized edge points has lower value of $\mathrm{d}$ and hence a high value of $R$. Therefore, the ideal edge map points have key importance in evaluating the performance of edge detectors using PFOM.

\section{IMPLEMENTATION AND EXPERIMENTAL RESULTS}

In order to perform quantitative performance evaluation, wellstructured synthetic rectangular-board image and Lena and Parrot image (figure 6(a)-(c)) of dimension 150x150, 512x512 and $768 \times 512$ (pixels), respectively has been considered to study the edge marking scheme of the detectors. The synthetic image drawn has well known edges and locations, which is generated using MATLAB Version 7.8.0.347 (R2009a). The lighter part in synthetic image has an intensity value of 255 and dark part has an intensity value of 0 , i.e. edge contrast is 255. The purpose of using three images is to make the quantitative analysis results more reliable and accurate. Figure 6(a) shows the synthetic image sample along with their edge locations in tabular form obtained from the five detectors, shown in table 1 .
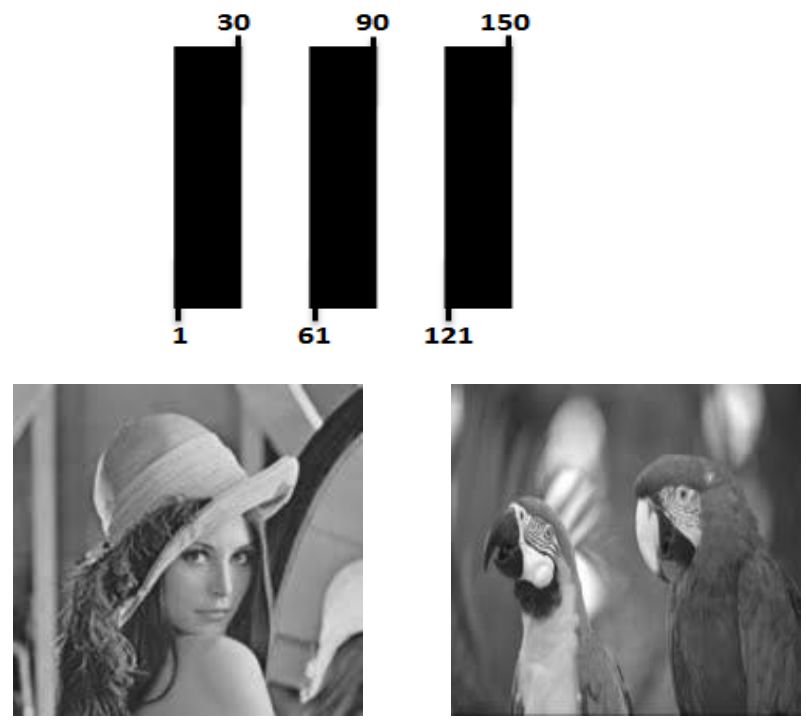

Fig 6: (a) Synthetic Image (150x150), (b) Lena image $(512 \times 512)$ and (c) Parrot Image $(768 \times 512)$

From table 1, it can be observed that Sobel and Prewitt detector marks the edge, after the intensity transient. This marking property is due to the fact that first order derivative based markers calculate the maximum rate of gradient per unit distance. High gradient value is only obtained after reaching into high-difference intensity region. Hence, edge is marked after the onset of the high-difference intensity region. Canny and Roberts detector marks the edge in a similar manner i.e. marking the edge before the onset of high-difference intensity region. Second order derivative based marker i.e. LoG produces double pixel thick edge. 
Table 1 Edge detection result for synthetic image, obtained from various edge detectors

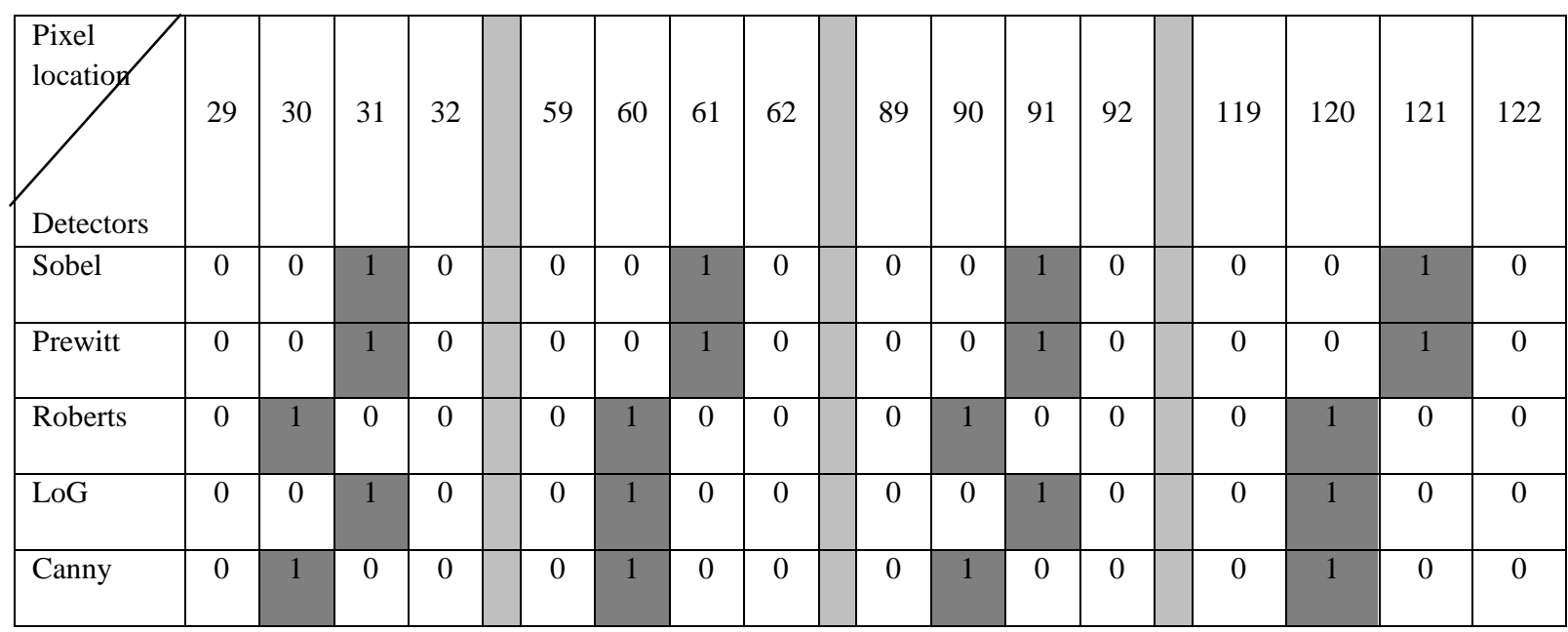

Therefore, to produce single pixel thick edge, global thresholding rule is applied on the edge map. Here, thresholding of the edge map by setting all positive zerocrossing values to white is done. After thresholding, it has been observed that LoG marks the edges in an alternate fashion (table 1).

PFOM is used to evaluate the performance of five edge detectors. Edge map obtained from Sobel, Prewitt, Roberts, LoG and Canny edge detectors has been considered commutatively as ideal edge maps, in order to calculate PFOM values for above considered five detectors. The PFOM values obtained for synthetic image, Lena and Parrot has been shown in table 3,4 and 5 , respectively.

Table 2 Numerical comparison in PFOM for synthetic image

\begin{tabular}{cccccc}
\hline & Sobel & Prewitt & Roberts & LoG & Canny \\
\hline Sobel & 100 & 100 & 90.90 & 95.37 & 95.37 \\
Prewitt & 100 & 100 & 90.90 & 95.37 & 95.37 \\
Roberts & 99.90 & 90.90 & 100 & 95.37 & 95.37 \\
LoG & 93.86 & 93.86 & 93.86 & 100 & 90.90 \\
Canny & 93.86 & 93.86 & 93.86 & 90.90 & 100 \\
\hline
\end{tabular}

Table 3 Numerical comparison in PFOM for Lena image

\begin{tabular}{cccccc}
\hline & Sobel & Prewitt & Roberts & LoG & Canny \\
\hline Sobel & 100 & 99.09 & 89.49 & 54.99 & 43.10 \\
Prewitt & 98.55 & 100 & 89.43 & 54.62 & 42.81 \\
Roberts & 88.36 & 88.95 & 100 & 51.12 & 39.83 \\
LoG & 70.20 & 69.63 & 63.94 & 100 & 70.01 \\
Canny & 57.67 & 57.01 & 52.64 & 80.05 & 100 \\
\hline
\end{tabular}

Table 4 Numerical comparison in PFOM for Parrot image

\begin{tabular}{cccccc}
\hline & Sobel & Prewitt & Roberts & LoG & Canny \\
\hline Sobel & 100 & 99.01 & 90.07 & 38.22 & 30.47 \\
Prewitt & 99.05 & 100 & 89.86 & 38.16 & 30.43 \\
& & & & & \\
Roberts & 87.97 & 87.94 & 100 & 35.28 & 27.99 \\
LoG & 51.25 & 50.64 & 44.87 & 100 & 69.00 \\
Canny & 41.39 & 40.91 & 36.10 & 76.91 & 100 \\
\hline
\end{tabular}

From the above discussion, results obtained on synthetic image, markers can be classified under three categories depending upon their marking scheme.

Pre-markers: This class of edge detectors marks an edge before high-difference intensity region e.g. Roberts and Canny edge detector.

Post-markers: These edge detectors mark an edge after highdifference intensity region e.g. Sobel and Prewitt edge detector.

Mixed-marker: As the name suggests, this class of marker mark an edge in an alternate manner (after thresholding, observed from the synthetic image) as in LoG detector.

In order to calculate PFOM, ideal edge map is to be necessarily defined. However, in the Pratt's model, ideal map is unclear therefore; edge map obtained from Sobel, Prewitt, Roberts, LoG and canny edge detector has been considered commutatively as an ideal edge map for the calculation of PFOM values. From table 2, it can be easily observed that same class of ideal edge map and detected edge map results in high PFOM values. On the other hand, different class ideal edge map and detected edge map results in lower PFOM values due to the fact that both the edge map differ in localization. As a result, Ideal edge map from different class penalizes for non-localized edges resulting in increased difference $d$ and hence reducing the value of rating factor $R$. 
Therefore, it is proposed to use ideal edge map from same class of detector, in order to calculate correct PFOM values.

A similar observation has also been made with the PFOM values for Lena and Parrot images, shown in table 3 and 4, respectively which strongly supports the above proposed edge detector classification.

\section{CONCLUSION}

In this paper, we presented a study on edge marking scheme of various standard edge detectors viz. Sobel, Prewitt, Roberts, LoG and Canny. We propose three types of edge markers, namely pre-marker, post-marker and mixed-marker, depending upon their marking schemes. Experimental results obtained for Lena, Parrot and synthetic image provides convincing results, to establish above explained edge detector classification.

Experimental results based on quantitative evaluation using PFOM, suggests considering ideal edge map from the same class as of actual edge detector, in order to calculate correct PFOM values. Considering ideal edge map from other class penalizes for non-localized edges, resulting into incorrect PFOM values.

\section{ACKNOWLEDGMENTS}

The authors thankfully acknowledge the kind inspiration provided by Prof. P. K. Bose, Director, National Institute of Technology, Agartala. Ms. Shaifali Pande and Mr. Vivek Singh Bhadouria acknowledge the fellowship provided by National Institute of Technology, Agartala for the research work.

\section{REFERENCES}

[1] Frei, W. and Chen, C. 1977. Fast Boundary Detection: A Generalization and New Algorithm. IEEE Trans. Computers C-26 (October 1977), 988-998.

[2] Gonzalez, R. C. and Woods, R. E. 2002. Digital Image Processing. Second ed. Pearson. Singapore.
[3] Pratt, W. K. 1978. Digital Image Processing. Wiley. New York.

[4] Roberts, L. G. 1965. Machine Perception of ThreeDimensional Solids. Optical and Electro-Optical Information Processing. Tippet. J. T. (ed.) MIT Press, Cambridge, Mass.

[5] Canny, J. 1986. A Computational Approach for Edge Detection. IEEE Trans. Pattern Anal. Machine Intell. 8(6), 679-698.

[6] Canny, J. 1983 Finding Edges and Lines in Image. Master's thesis. MIT.

[7] Maini, R. and Aggarwal, 2009. H. Study and Comparison of Various Image Edge Detection Techniques. Inter. Journal of Image Processing. 3(1). 111.

[8] Gunn, S. R. 1998. Edge Detection Error in the Discrete Laplacian of Gaussian. Proc. 1988 Inter. Conference on Image Processing. 2, 515-519.

[9] Gunn, S. R. 1999. On the Discrete Representation of the Laplacian of Gaussian. Pattern Recognition. 32(8). 14631472.

[10] Prewitt, J. M. S. 1970. Object Enhancement and Extraction. Picture Processing and Psychopictorics, Lipkin, B. S. and Rosenfeld, A. (Eds.). Academic Press, New York.

[11] Heath, M., Sarkar, S., Sanocki, T. and Bowyer, K. W. 1998. Comparison of Edge Detectors: A Methodology and Initial Study. Computer Vision and Image Understanding, 69(1), 38-54.

[12] Abdou, I. E. and Pratt, W. K. 1979. Quantitative design and evaluation of enhancement/thresholding edge detectors. Proc. of the IEEE. 67(5). 753-763. 